\title{
RELATING THE BOTTOM PRESSURE AND THE SURFACE ELEVATION IN THE WATER WAVE PROBLEM
}

\author{
B. DECONINCK \\ Department of Applied Mathematics, University of Washington \\ Seattle, WA 98195-2420, USA \\ bernard@amath.washington.edu \\ K. L. OLIVERAS \\ Department of Mathematics, Seattle University \\ Seattle, WA 98122, USA \\ oliverak@seattleu.edu \\ V. VASAN \\ Department of Mathematics, Pennsylvania State University \\ University Park, PA 16802, USA \\ vasan@math.psu.edu \\ Received (Day Month Year) \\ Revised (Day Month Year) \\ Accepted (Day Month Year)
}

\begin{abstract}
An overview is presented of recent progress on the relation between the pressure at the bottom of the flat water bed and the elevation of the free water boundary within the context of the onedimensional, irrotational water wave problem. We present five different approaches to this problem. All are compared to (1) numerical data for Stokes waves, one-dimensional traveling wave solutions of the full irrotational water wave problem, and (2) experimental data for high-amplitude waves in a long, narrow (i.e., one-dimensional) wave tank.
\end{abstract}

Keywords: water waves; pressure; review.

2000 Mathematics Subject Classification: 35Q31, 76B15, 76B07

\section{Introduction}

The dynamics of an irrotational, inviscid free-surface water wave is accurately modeled by the Euler equations. In the case of a one-dimensional surface, these equations are

$$
\begin{aligned}
\phi_{x x}+\phi_{z z} & =0, & & (x, z) \in D, \\
\phi_{z} & =0, & & z=-h, \\
\eta_{t}+\eta_{x} \phi_{x} & =\phi_{z}, & & z=\eta(x, t),
\end{aligned}
$$




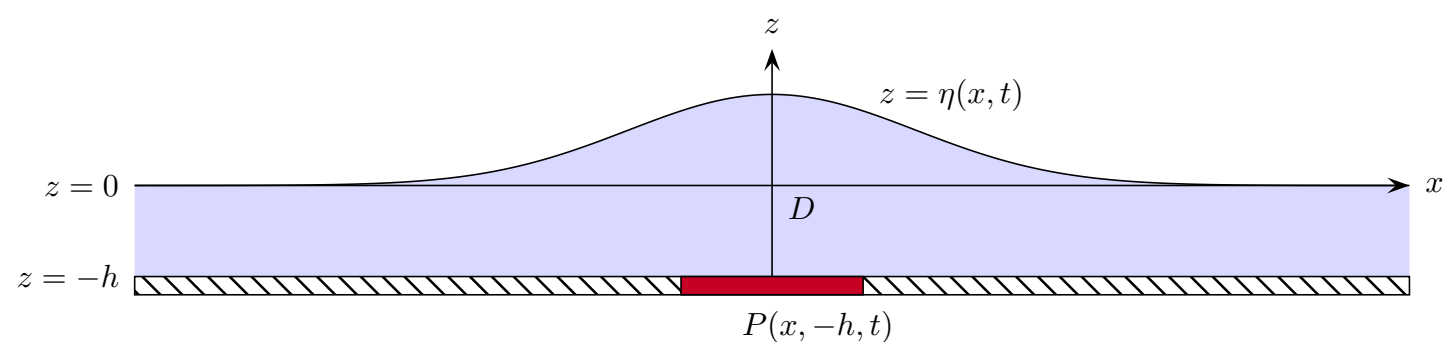

Fig. 1. The fluid domain $D$ for the water wave problem. An idealized pressure sensor is indicated at the bottom. In all models discussed the pressure measurement is assumed to be a point measurement.

$$
\phi_{t}+\frac{1}{2}\left(\phi_{x}^{2}+\phi_{z}^{2}\right)+g \eta=0, \quad z=\eta(x, t)
$$

where $x$ and $z$ are the horizontal and vertical coordinate, respectively, see Figure $1 ; z=$ $\eta(x, t)$ is the free top boundary and $\phi(x, z, t)$ is the velocity potential. Indices are used to denote partial derivatives. Further, $g$ is the acceleration due to gravity and $h$ is the average depth of the fluid.

A quantity of significant physical importance is the pressure $P(x, z, t)$ in the fluid. This quantity does not appear in the Euler equations. Instead, it is obtained from the Bernoulli equation

$$
\phi_{t}+\frac{1}{2}\left(\phi_{x}^{2}+\phi_{z}^{2}\right)+g z+\frac{P(x, z, t)}{\rho}=0, \quad-h \leq z \leq \eta(x, t)
$$

which allows one to find the pressure once (1a-d) is solved.

However, from a physical perspective one may wish to ask a different question: is it possible to recover the surface elevation $\eta(x, t)$ from knowledge of the pressure, especially when measured at the bottom $z=-h$ ? This problem is of paramount importance for experimentalists and field practitioners, for whom direct measurement of the surface elevation is difficult. Instead, the surface elevation is often inferred from measurements of the bottom pressure, see e.g. [1], [2], [3], [4], [5], [6]. For instance, the predictions made by the Pacific Tsunami Warning Center [7] are determined this way. The pressure is not only of physical importance: it is relevant for the mathematical study of the Euler equations. Its qualitative properties play a central role in understanding properties of irrotational traveling water waves (so-called Stokes waves) such as in showing that their free surface is the graph of a function [8,9], and in discerning the patterns of particle paths beneath them [10-12].

These considerations prompt us to investigate (1a-d) and (2) in a different way, where the bottom pressure is considered as input, and the goal is to recover the surface quantities $\phi(x, \eta(x, t), t)$ and, especially, $\eta(x, t)$. In addition, we impose that the solution of (1a-d, 2) are stationary in a frame of reference moving with constant velocity (in other words, we restrict ourselves to Stokes wave solutions) and one dimensional. When comparing with experimental data in Section 4 this can no longer be justified, and we explore the extent to which the different methods for surface reconstruction still provide accurate results. 


\section{Relating the bottom pressure and the surface elevation}

The problem of relating the water surface elevation to measurements at the flat fluid bed has received much attention recently. In this section we review five relationships between the bottom pressure and the surface elevation. Three of these are the result of recent work. In the following sections we compare these relationships against numerical and experimental data.

- The oldest and perhaps most commonly used reconstruction formula is that obtaining using a Hydrostatic Approximation (Archimedes' relation) [13, 14]:

$$
\eta(x, t)=\frac{P(x,-h, t)}{\rho g}-h .
$$

As mentioned in the introduction, the hydrostatic approximation is used, for instance, in open-ocean buoys employed for tsunami detection, see [7]. Despite the limitations of a hydrostatic approximation, its application in a shallow-water regime (e.g., tsunamis) are remarkably accurate. A comparison with field data is not included below but one easily verifies, using pressure data available from [7], for instance, that the four formulations below simply replicate the surface obtained using (1).

- The Transfer Function approach is obtained by linearizing the equations of motion around quiescent water, and using Fourier transforms to solve the resulting linear constant coefficient equations, similar to how one derives the dispersion relation for the water wave problem. This results in a linear relationship between the Fourier transforms $\mathcal{F}$ of the dynamical part of the pressure and the elevation of the surface $[13-16]$ :

$$
\mathcal{F}\{\eta(x, t)\}(k)=\cosh (k h) \mathcal{F}\{p(x, t) / g\}(k) .
$$

Here $p(x, t)=(P(x,-h, t)-\rho g h) / \rho$ is the dynamic (or non-static) part of the pressure $P(x, z, t)$ evaluated at the bottom of the fluid $z=-h$, scaled by the fluid density $\rho$. In this relationship, $\eta$ and $p$ are regarded as functions of the spatial coordinate $x$, with parametric dependence on time $t$. Since the formula is the result of linearizing (1a-d, 2), one can only expect good agreement for waves of small amplitude. In particular, the reconstruction of waves that do not have a linear analogue (like solitary waves, which also have a broad spectrum) cannot be assumed to be accurate using (2). In contrast to the other relations presented here, (2) is not restricted to Stokes Waves. In measurements, time series are more common than spatial series. The formula (2) is easily modified to accommodate this, using temporal instead of spatial Fourier transforms. This results in extra factors of the wave speed $c(k)$, not necessarily constant.

Both approaches above rely on linear theory. Until the work of Escher \& Schlurmann [15], no consideration was given to the importance of nonlinear effects in the pressure-surface reconstruction problem. Such effects are expected to be especially significant for highamplitude shallow-water waves or for waves in the surf zone (see $[2,6,17]$, for instance). 
Since nonlinear effects are not captured by the linear transfer function (2), different modifications of (2) have been proposed, see [3,4,6] or [17] and [18]. See Bishop \& Donelan [2] for a comparison of these modified transfer function approaches.

Since 2008, much progress has been made in understanding the qualitative and quantitative properties of the pressure in the water wave problem, accounting for all nonlinear effects. Constantin \& Strauss [19] examine different properties and relations between the pressure and the surface elevation qualitatively. They do not present a reconstruction method to accurately determine one function in terms of the other, but the mathematical properties they uncover are revisited and verified in an experimental setting by Constantin, Escher \& Hsu [20].

- The first nonlinear reconstruction method was introduced in [21,22] and is based on a fully nonlinear nonlocal implicit relationship between the surface elevation and bottom pressure. The Nonlocal Relationship is obtained from (1a-d, 2) with a traveling wave assumption but without any other approximation. In order to recover the surface elevation from the pressure measurements one solves

$$
\sqrt{\frac{c^{2}-2 g \eta}{1+\eta_{\xi}^{2}}}=\frac{1}{2 \pi} \int_{-\infty}^{\infty} e^{i k \xi} \cosh (k(\eta+h)) \mathcal{F}\left\{\sqrt{c^{2}-2 p}\right\}(k) d k
$$

for $\eta(\xi)=\eta(x-c t)$ given $p(\xi)=p(x-c t)$. Here $c$ is the speed of the traveling wave. Using an Implicit Function Theorem argument, given $c$ and $p$, it is shown in [22] that (3) has a solution, at least for waves of sufficiently small amplitude. Using numerical and experimental comparisons, excellent agreement between $\eta(\xi)$ and the true surface elevation was obtained. These comparisons were carried out well beyond the small amplitude regime, and with waves that were not necessarily traveling. The insensitivity of the results obtained with regard to the parameter $c$ seems to be one of the advantages of this relationship. This is explored further below.

As given above, (3) applies to solitary waves. An equivalent relation for periodic solutions is easily derived, see [22]. It is this periodic equivalent that is used below when comparing with numerical or experimental data.

- As demonstrated in [22], the nonlocal relationship (3) may be used to derive several asymptotic relationships. One such approximation is the Renormalized Transfer Function given by

$$
\eta=\frac{\mathcal{F}^{-1}\{\hat{p}(k) \cosh (k h)\} / g}{1-\mathcal{F}^{-1}\{\hat{p}(k) k \sinh (k h)\} / g},
$$

where $\hat{p}(k)=\mathcal{F}\{p(\xi)\}(k)$. This relation may be viewed as an improvement to the traditional transfer function (2) and is valid for small amplitude waves. As noted in [22] (or, see below), this approximation is a good compromise between accuracy and computational efficiency, even for waves of large amplitude. Unlike the fully nonlinear model (3), the Renormalized Transfer Function does not require knowledge 
of the wave speed $c$ explicitly, although the traveling wave assumption is made in its derivation.

- Recently, Constantin [23] derived a fully nonlinear relation that allows for the recovery of a traveling solitary wave profile from measurements of the bottom pressure. In contrast to the methods mentioned above, the recovery formula holds for solitary wave profiles only. An analogue for periodic waves is not known. It is given in parametric form as

$$
\begin{aligned}
& \xi(q)=q+\int_{-\infty}^{q} \mathcal{F}^{-1}\left\{\cosh (k h) \mathcal{F}\left\{\frac{c}{\sqrt{c^{2}-2 p}}-1\right\}(k)\right\}(s) d s, \\
& \eta(q)=\mathcal{F}^{-1}\left\{\frac{\sinh (k h)}{k} \mathcal{F}\left\{\frac{c}{\sqrt{c^{2}-2 p}}-1\right\}(k)\right\}(q) .
\end{aligned}
$$

We refer to this relation as the Explicit Solitary Wave Reconstruction. Formula (5) is obtained without approximation from (1a-d, 2) with the imposition of a traveling wave assumption, posed on the infinite line. It is amazing that the relationship between pressure and surface elevation is explicit: from the knowledge of $c$ and $p$, the graph of $\eta(\xi)$ is obtained immediately.

\section{Numerical Comparisons of the Different Approaches}

In this section, we compare the reconstructed surface using the various relationships discussed in the previous section using numerical data. We investigate the accuracy of the reconstruction and the sensitive dependence, if any, on the parameter $c$ of the different reconstructions.

\subsection{Reconstruction using Numerical Data}

The five reconstruction methods introduced above are compared with periodic Stokes wave data generated from the Euler equations (1a-d), using the methods discussed in [24]. This comparison is an extension of that in [22], where the Explicit Solitary Wave Reconstruction was not included. Without loss of generality, we assume the waves have period $2 \pi$. It is well known that once the period and the depth $h$ are fixed, there exists a single branch of Stokes wave solutions bifurcating away from the trivial, quiescent solution (see [24] and many of the references therein). We start with a solution on this bifurcation curve, i.e., we prescribe $c$ and a $2 \pi$-periodic Stokes wave. Using (3), we solve for the pressure at the bottom by equating the Fourier coefficients on both its left- and right-hand side (for $k=-N \ldots N$, $N$ a chosen cut-off value). Using the truncated algebraic system of equations, we solve the linear system for the Fourier series representation of the term $\sqrt{c^{2}-2 p}$. This allows for the direct solution for $p(\xi)$ in terms of the Stokes wave data set $\mathcal{S}=\left(\eta_{\text {true }}(\xi), c_{\text {true }}\right)$.

Having obtained the pressure underneath a Stokes wave, we use the various relations presented in Section 2 to reconstruct the surface elevation. As a validation, we expect that the reconstruction (3) using the Nonlocal Relation should be accurate to machine precision since we are using a nonlinear solver to invert the transformation that generated the pressure data. 
Using the non-dimensional parameters $h=.1, g=1, \rho=1$ and $L=2 \pi$, the reconstruction using the different relations is shown in Figure 2. The top panel shows the reconstruction of a solution of small amplitude. It appears that all reconstruction methods are in good agreement. This is somewhat surprising for the Explicit Solitary Wave Reconstruction using (5), which is valid only for solitary wave solutions. The small-amplitude solution is not near the solitary wave regime. In order to compare (5) with periodic data, its range of $x$ values is simply truncated. Increasing the amplitude $\left(=\left(\eta_{\max }-\eta_{\min }\right) / 2\right)$ of the true solution $\eta_{\text {true }}(\xi)$ shows differences between the reconstructions, even though the amplitude is less than $35 \%$ of the limiting wave height as given by [25]. In particular, the Nonlocal Relation (3) provides near-perfect agreement, as it should. Its reconstruction cannot be distinguished from the true surface elevation. On the other hand, the results using the Hydrostatic Approximation (1) show significant errors. This error, at least at the peak of the wave, is roughly halved by using the Transfer Function (2). The error at the peak is decreased more by using the Explicit Solitary Wave Reconstruction (5), although the Transfer Function does better elsewhere. A significant improvement is obtained from the Renormalized Transfer Function (4), as is quantified in the next section.

We should emphasize that the comparison of the Explicit Solitary Wave Reconstruction (5) with the other approaches on the numerical data used here is unfair: the numerical data is periodic, whereas (5) applies to solitary waves. Obtaining solitary wave solutions of the Euler equations (1a-d) is more complex than computing periodic solutions (see [26], for instance), and it is not pursued here. Nevertheless, (5) provides an accurate reconstruction, even outside its regime of validity.

\subsection{Sensitivity with respect to $c$}

In this section we quantify the errors observed above, specifically as a function of changing c. In an experimental or field setting, measuring the velocity $c$ is not trivial and one is tempted to use an educated guess. As demonstrated in [22], many of the reconstruction methods display little or no $c$ dependence. This statement is trivial for the Hydrostatic Approximation (1), and for the Transfer and Renormalized Transfer Functions (2, 4), but not so for the Nonlocal Relation or for other reconstructions used in [22]. In addition to quantifying errors in the reconstruction, we wish to examine the sensitivity with respect to $c$ of the Explicit Solitary Wave Reconstruction formula (5).

We quantify the relative error in the surface reconstruction using the infinity norm:

$$
\text { Relative Error }=\frac{\left\|\eta_{t r u e}-\eta_{r}\right\|_{\infty}}{\left\|\eta_{\text {true }}\right\|_{\infty}}
$$

where $\eta_{r}$ represents the reconstructed surface elevation. Figure 3 displays this Relative Error for the reconstruction of a Stokes wave of amplitude 0.0056, with parameters as in Figure 2. The error is presented as a function of varying $c$, ranging from a first guess of $\sqrt{g h}=0.316$, over the correct value of 0.32774 , up to an overestimate of more than 0.034. Unsurprisingly, the Hydrostatic Approximation (1) shows the largest error, while not depending on $c$. Similarly, the Transfer Function (2) and the Renormalized Transfer Function (4) have no $c$ dependence, and result in the same error for this solution. The lowest error is obtained using the Nonlocal Relation (3). In agreement with [22], this reconstruction exhibits little 

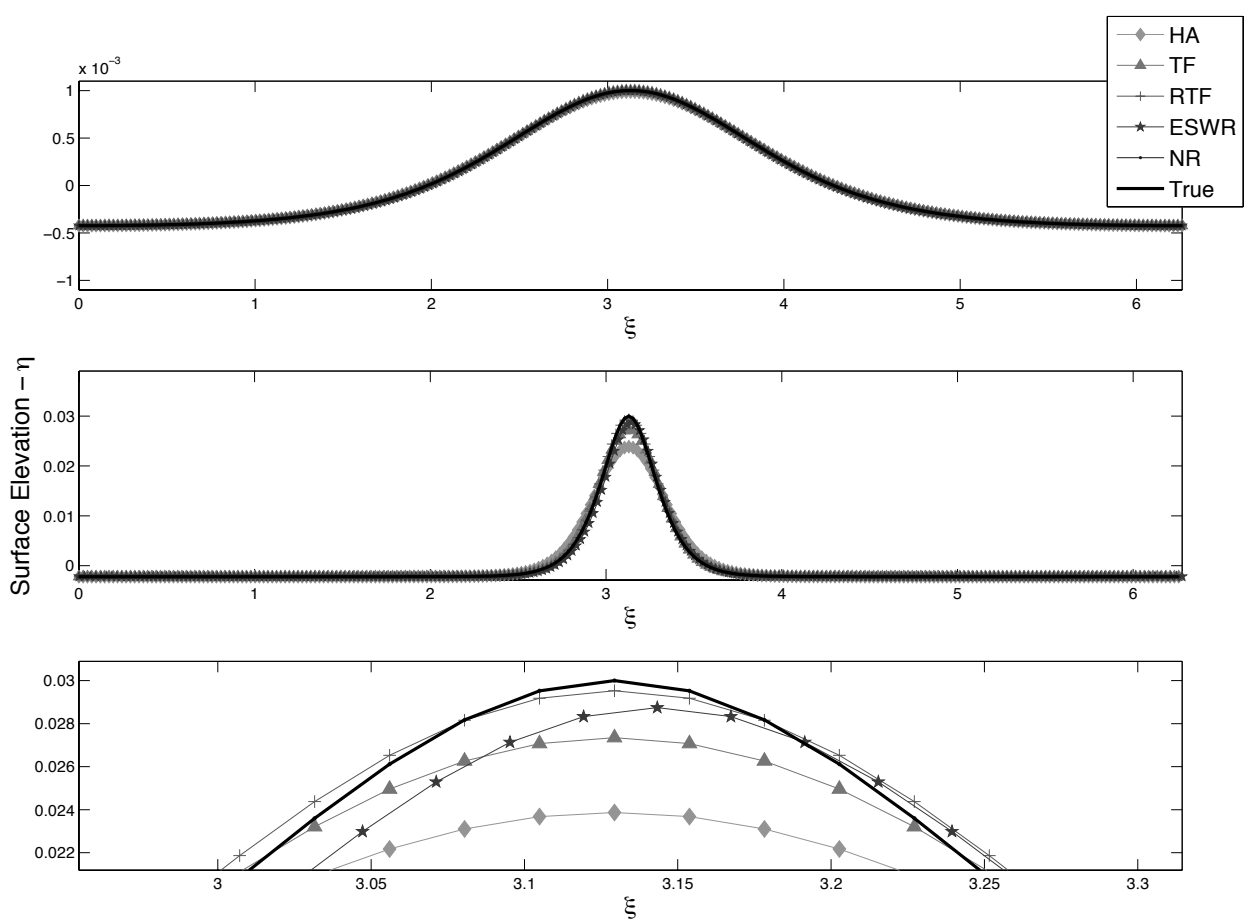

Fig. 2. Reconstruction of the surface elevation from pressure data using numerical data with $h=0.1$, $g=1, \rho=1$ and $L=2 \pi$. Shown are the Hydrostatic Approximation (HA), the Transfer Function (TF), the Nonlocal Relation (NR), the Renormalized Transfer Function (RTF), and the Explicit Solitary Wave Reconstruction (ESWR). The surface elevation amplitude for the top panel is 0.0007 , while for the middle panel it is 0.0161 . The bottom panel is a zoom of the peak area of the middle panel.

or no dependence on $c$. A more precise statement is that the $c$ dependence of (3) is not visible in Figure 3, which has a vertical scale dominated by the difference in error between the Hydrostatic Approximation (1) and round-off error, attained using Nonlocal Relation (3).

The Explicit Solitary Wave Reconstruction (5) displays more sensitive dependence on the parameter $c$. Indeed, it appears that the choice of the correct value of $c$ is crucial in order to minimize the error of the reconstruction. In fact, the minimal error is achieved for a value of $c$ that slightly exceeds the correct value. Presumably, this is due to the fact that we are comparing a solitary wave reconstruction with a periodic solution.

\section{Physical Experiments}

Using the various formulae discussed in Section 2, we reconstruct the surface elevation from pressure data obtained from ten laboratory experiments performed at Penn State's Pritchard Fluid Mechanics Laboratory. The experimental facility consists of the wave channel and water, the wavemaker, bottom pressure transducers, and a surface displacement measurement system. The wavetank is $50 \mathrm{ft}$ long, $10 \mathrm{in}$ wide and $1 \mathrm{ft}$ deep and was filled with tap water to a depth of $h$, as listed in Table 1 . Waves are generated using a paddle programmed with a KdV soliton solution. The pressure at the bottom of the fluid domain and 


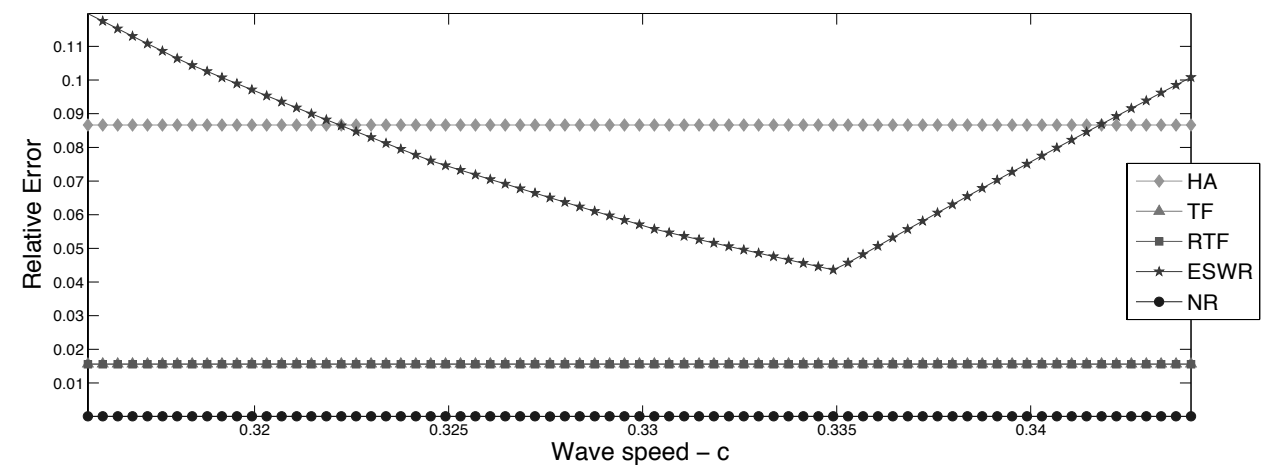

Fig. 3. Plot of the error in the reconstructed surface elevation $\eta_{r}$ as a function of the amplitude of $\eta_{\text {true }}$ using an approximation for the wave-speed $c$.

the displacement of the air-water interface are measured simultaneously at some distance from the wave generator. See [22] for more details of the experimental set-up.

From the experiments, we obtain a time-series of data for the pressure at the bottom of the tank, and the surface elevation directly above the pressure sensor. Using a conversion factor of $\sqrt{g h}$, this data is converted from a time series to a spatial series. Using the pressure data measured from the physical experiments, we reconstruct the surface elevation using the same methods illustrated in the previous section with the additional step of prefiltering the data to remove any high-frequency noise using a low-pass filter. The cut-off frequency is chosen so that the shape of the filtered data preserves the same general characteristics as the unfiltered data. More details are found in [22].

Using the filtered pressure data and an estimated wave speed $c$ (again taken to be $c \approx \sqrt{g h}$, as the simplest guess), we reconstruct the surface elevation numerically. A visual comparison of the different reconstructions is presented in Figure 4. Focusing on the peak height, it appears all methods perform well, but some differences are clear: the Hydrostatic Approximation (1) provides the worst reconstruction, while the Nonlocal Relation (3) results in the most accurate peak height reconstruction.

More quantitatively, for a total of ten experiments (see [22]), we reconstruct the surface elevation in the same manner and compare the results with the measured surface elevation. Table 1 displays the results of this comparison, using the relative error in the peak height as a metric:

$$
\text { Relative Error in peak height }=\left|\frac{\left\|\eta_{\text {true }}\right\|_{\infty}-\left\|\eta_{r}\right\|_{\infty}}{\left\|\eta_{\text {true }}\right\|_{\infty}}\right|
$$

We use this metric since we want to focus on capturing the wave height, without getting lost in low-amplitude noise and the interference of nearby waves, as is visible in Figure 4. Further, it is expected that the maximal difference in the vicinity of the wave peak occurs at the wave peak, making (1) equivalent to (1), up to additional windowing to an area centered around the peak. Some of the results in Table 1 are repeated from [22].

As expected, the Hydrostatic Approximation (HA) performs the worst, with errors of around 20\%. The error is reduced significantly using the Transfer Function (TF). Compared 


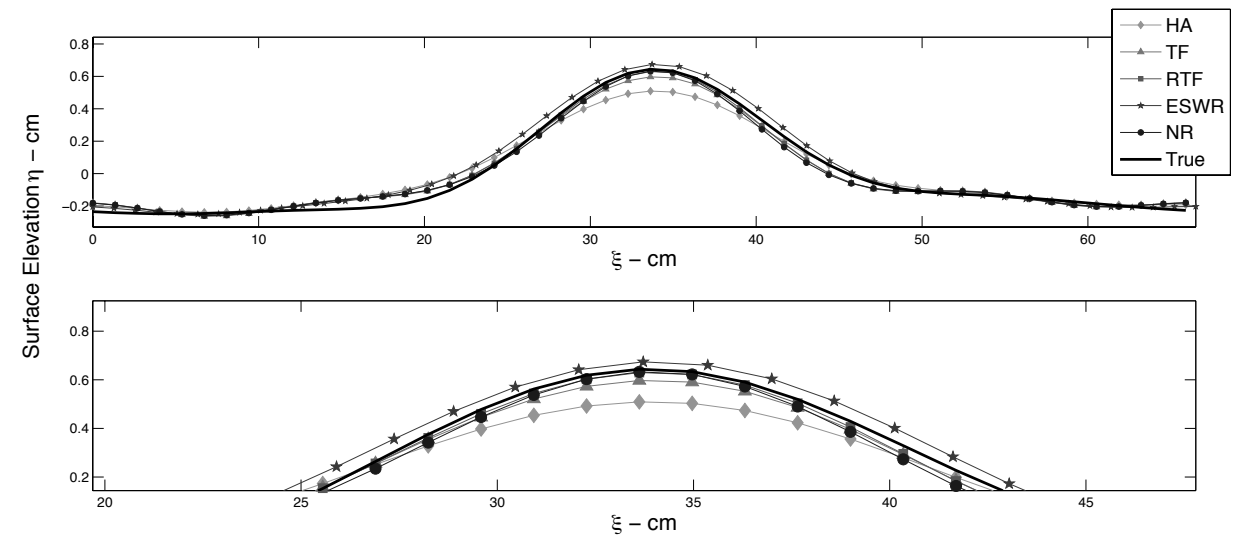

Fig. 4. Comparisons of the reconstructed surface elevation with wave-tank surface-height measurements with $h=3.55 \mathrm{~cm}$, Experiment \#7.

Table 1. Error calculated comparing peak wave heights.

\begin{tabular}{l|l||c|c|c|c|c} 
Exp \# & Depth $(\mathrm{cm})$ & HA & TF & ESWR & RTF & NR \\
\hline \hline 1 & $5.05 \mathrm{~cm}$ & $22.29 \%$ & $6.51 \%$ & $3.50 \%$ & $0.45 \%$ & $0.20 \%$ \\
2 & $5.05 \mathrm{~cm}$ & $24.66 \%$ & $8.05 \%$ & $3.67 \%$ & $0.56 \%$ & $0.01 \%$ \\
3 & $5.05 \mathrm{~cm}$ & $23.56 \%$ & $7.75 \%$ & $6.62 \%$ & $0.41 \%$ & $0.18 \%$ \\
4 & $5.05 \mathrm{~cm}$ & $21.98 \%$ & $7.18 \%$ & $6.47 \%$ & $0.89 \%$ & $0.66 \%$ \\
5 & $5.05 \mathrm{~cm}$ & $22.00 \%$ & $6.63 \%$ & $6.28 \%$ & $0.05 \%$ & $0.03 \%$ \\
6 & $3.55 \mathrm{~cm}$ & $18.11 \%$ & $5.21 \%$ & $8.67 \%$ & $0.43 \%$ & $0.36 \%$ \\
7 & $3.55 \mathrm{~cm}$ & $20.81 \%$ & $7.04 \%$ & $6.36 \%$ & $1.95 \%$ & $1.52 \%$ \\
8 & $4.10 \mathrm{~cm}$ & $21.59 \%$ & $8.39 \%$ & $15.60 \%$ & $1.73 \%$ & $0.93 \%$ \\
9 & $4.10 \mathrm{~cm}$ & $22.13 \%$ & $7.65 \%$ & $15.74 \%$ & $0.31 \%$ & $0.05 \%$ \\
10 & $4.10 \mathrm{~cm}$ & $23.32 \%$ & $9.25 \%$ & $13.18 \%$ & $2.44 \%$ & $2.13 \%$
\end{tabular}

to the Transfer Function, the Explicit Solitary Wave Reconstruction (ESWR) results in a better peak reconstruction for Experiments \#1-\#5, \#7, but it is worse for the other experiments, by quite a big margin. Once again, our use of techniques for periodic waves (such as Fast Fourier Transforms) as opposed to solitary waves could be (at least partially) at the source of this discrepancy. On the other hand, any comparison with experimental data is likely to approximate a solitary wave with a large-period periodic wave. Another possible explanation is the sensitivity of ESWR (5) with respect to $c$, as demonstrated in the previous section, see also below. This is especially relevant given that we have used the simple approximation $c=\sqrt{g h}$ for all comparisons. Lastly, the Renormalized Transfer Function (4) and the Nonlocal Relation (3) result in the most accurate peak reconstruction, as already reported in [22].

Lastly, we examine the effect of changing the value of $c$ in the reconstruction of peak heigth of the experimental surface elevation. Table 2 summarizes our findings: we vary the value of $c$ from $0.95 \sqrt{g h}$ to $1.8 \sqrt{g h}$ (a "correct" value of $c$ in this experimental setting is not known, if one exists). Of course, the Renormalized Transfer Function (RTF) is not sensitive 
Table 2. Peak Height Error as a function of $c$ for $h=5.05 \mathrm{~cm}$, Experiment \#1.

\begin{tabular}{r|c|c|c}
$\%$ deviation & RTF & ESWR & NR \\
\hline $.95 \sqrt{g h}$ & $0.41 \%$ & $18.42 \%$ & $0.29 \%$ \\
$\sqrt{g h}$ & $0.41 \%$ & $3.54 \%$ & $0.19 \%$ \\
$1.05 \sqrt{g h}$ & $0.41 \%$ & $8.49 \%$ & $0.13 \%$ \\
$1.1 \sqrt{g h}$ & $0.41 \%$ & $18.41 \%$ & $0.09 \%$ \\
$1.6 \sqrt{g h}$ & $0.41 \%$ & $65.17 \%$ & $0.07 \%$ \\
$1.7 \sqrt{g h}$ & $0.41 \%$ & $69.40 \%$ & $0.10 \%$ \\
$1.8 \sqrt{g h}$ & $0.41 \%$ & $72.85 \%$ & $0.15 \%$
\end{tabular}

to changes in $c$. The results obtained using both the Explicit Solitary Wave Reconstruction (ESWR) and the Nonlocal Relation (NR) do depend on $c$, but much more so for ESWR than for NR, as expected from the results in the previous section.

\section{Conclusion}

We have presented an overview of different approaches to reconstruct the surface elevation from pressure measurements at the flat bottom of an irrotational, inviscous fluid under the influence of gravity, as modeled by Euler's Equations (1a-d). Some of these approaches result in approximate reconstructions (the Hydrostatic Approximation (1), the Transfer Function (2), the Renormalized Transfer Function (4)), while others give exact reconstructions within the context of the Euler equations (the Nonlocal Relation (3), the Explicit Solitary Wave Reconstruction (5)).

We have applied these approaches to the problem of surface reconstruction using numerical data for Stokes wave solutions of the Euler Equations. In this case the velocity parameter $c$ is known, and all method except the Hydrostatic Approximation result in accurate reconstructions, with the nonlinear methods outperforming the linear ones. Given the uncertainty on $c$ in an experimental setting, it is desirable that the reconstruction method used has low sensitivity with respect to this parameter. This is examined numerically, leading to the conclusion that all methods except the Explicit Solitary Wave Reconstruction (5) display this desired low sensitivity. This is surprising for the Nonlocal Relation (3).

The consequences of this are especially felt when reconstructing the surface peak height using experimental data. In this case, an educated guess $(c \sim \sqrt{g h})$ is used for the velocity parameter, resulting in highly accurate peak reconstructions using the Nonlocal Relation (3) and the Renormalized Transfer Function (4). Both methods combine the effect of nonlinearity and low sensitivity with respect to $c$, enhancing their applicability in real-world-settings. Due to the explicit nature of its reconstruction (requiring the computation of a mere three Fourier transforms) the Renormalized Transfer Function seems to present the best balance between computational efficiency and reconstruction accuracy.

\section{Acknowledgements}

BD and VV acknowledge support from the National Science Foundation under grant NSFDMS-1008001. Any opinions, findings, and conclusions or recommendations expressed in 
this material are those of the authors and do not necessarily reflect the views of the funding sources.

\section{References}

[1] A. Baquerizo and M. A. Losada, Transfer function between wave height and wave pressure for progressive waves, Coastal Engineering 24 (1995) 351-353.

[2] C. T. Bishop and M. A. Donelan, Measuring waves with pressure transducers, Coastal Engineering 11 (1987) 309-328.

[3] Y.-Y. Kuo and J.-F. Chiu, Transfer function between the wave height and wave pressure for progressive waves, Coastal Engineering 23 (1994) 81-93.

[4] Y.-Y. Kuo and J.-F. Chiu, Transfer Function between wave height and wave pressure for progressive waves: reply to the comments of A. Baquerizo and M.A. Losada, Coastal Engineering 24 (1995) 355-356.

[5] J.-C. Tsai and C.-H. Tsai, Wave measurements by pressure transducers using artificial neural networks, Ocean Engineering 36 (2009) 1149-1157.

[6] C.-H. Tsai, M. C. Huang, F. J. Young, Y. C. Lin and H. W. Li, On the recovery of surface wave by pressure transfer function, Ocean Engineering 32 (2005) 1247-1259.

[7] National Oceanic and Atmospheric Administration, Pacific Tsunami Warning Center http://ptwc. weather.gov/.

[8] E. R. Spielvogel, A variational principle for waves of infinite depth, Arch. Rational Mech. Anal. 39 (1970) 189-205.

[9] E. Varvaruca, Singularities of Bernoulli free boundaries, Comm. Part. Diff. Eqns. 31 (2006) $1451-1477$.

[10] A. Constantin, The trajectories of particles in Stokes waves, Invent. Math. 166 (2006) 523-535.

[11] A. Constantin and J. Escher, Particle trajectories in solitary water waves, Bull. Amer. Math. Soc. 44 (2007) 423-431.

[12] A. Constantin, On the particle paths in solitary water waves, Quart. Appl. Math. 68 (2010) $81-90$.

[13] I. M. Cohen and P. K. Kundu, Fluid Mechanics (Academic Press, 2004).

[14] R. G. Dean and R. A. Dalrymple, Water Wave Mechanics for Engineers and Scientists (World Scientific, Singapore, 2010).

[15] J. Escher and T. Schlurmann, On the recovery of the free surface from the pressure within periodic traveling water waves, J. Nonlinear Math. Phys. 15 (2008) 50-57.

[16] A. B. Kennedy, R. Gravois, B. Zachry, R. Luettich, T. Whipple, R. Weaver, J. ReynoldsFleming, Q. J. Chen and R. Avissar, Rapidly installed temporary gauging for hurricane waves and surge, and application to Hurricane Gustav, Continental Shelf Research 30 (2010) 17431752 .

[17] A. O. Bergan, A. Torum and A. Traetteberg, Wave measurements by pressure type wave gauge, Proceedings of the 11th Coastal Engineering Conference ASCE (1968) 19-29.

[18] D. Y. Lee and H. Wang, Measurement of surface waves from subsurface gage, Proceedings of the 19th Coastal Engineering Conference ASCE (1984) 271-286.

[19] A. Constantin and W. Strauss, Pressure and trajectories beneath a Stokes wave, Comm. Pure Appl. Math. 53 (2010) 533-557.

[20] A. Constantin, J. Escher and H.-C. Hsu, Pressure beneath a solitary water wave: mathematical theory and experiments, Arch. Ration. Mech. Anal. 201 (2011) 251-269.

[21] B. Deconinck, D. Henderson, K. Oliveras and V. Vasan, Recovering the water-wave surface from pressure measurements, in Proc. 10th International Conference on Mathematical and Numerical Analysis of Waves (WAVES 2011), (PIMS, Vancouver, BC, 2011) pp. 699-703.

[22] K. Oliveras, V. Vasan, B. Deconinck and D. Henderson, Recovering the water-wave profile from pressure measurements, SIAM J. of Applied Mathematics 72 (2012) 897-918. 
12 B. Deconinck, K. L. Oliveras, V. Vasan

[23] A. Constantin, On the recovery of solitary wave profiles from pressure measurements, Journal of Fluid Mechanics FirstView (2012) 1-9.

[24] B. Deconinck and K. Oliveras, The instability of periodic surface gravity waves, J. Fluid Mech. 675 (2011) 141-167.

[25] E. D. Cokelet, Steep gravity waves in water of arbitrary uniform depth, Phil. Trans. Roy. Soc. London. Ser. A 286 (1977) 183-230.

[26] M. J. Ablowitz, A. S. Fokas and Z. H. Musslimani, On a new non-local formulation of water waves, J. Fluid Mech. 562 (2006) 313-343. 